\title{
Transtorno dismórfico corporal em dermatologia: diagnóstico, epidemiologia e aspectos clínicos ${ }^{*}$
}

\author{
Body dysmorphic disorder in dermatology: diagnosis, epidemiology \\ and clinical aspects
}

\author{
Luciana Archetti Conrado ${ }^{1}$
}

\begin{abstract}
Resumo: São cada vez mais frequentes as queixas cosméticas, uma vez que objetivam a perfeição das formas do corpo e da pele. Os dermatologistas são consultados para avaliar e tratar essas queixas. Sendo assim, é importante conhecer o Transtorno Dismórfico Corporal, inicialmente chamado de "dismorfofobia”, pouco estudado até recentemente. Esse transtorno é relativamente comum, por vezes, incapacitante, e envolve uma percepção distorcida da imagem corporal, caracterizada pela preocupação exagerada com um defeito imaginário na aparência ou com um mínimo defeito corporal presente. A maioria dos pacientes apresenta algum grau de prejuízo no funcionamento social e ocupacional, e como resultado de suas queixas obsessivas com a aparência, podem desenvolver comportamentos compulsivos, e, em casos mais graves, há risco de suicídio. O nível de crença é prejudicado, visto que não reconhecem o seu defeito como mínimo ou inexistente e, frequentemente, procuram tratamentos cosméticos para um transtorno psíquico. A prevalência do transtorno, na população geral, é de 1 a $2 \%$ e, em pacientes dermatológicos e de cirurgia cosmética, de 2,9 a 16\%. Considerando a alta prevalência do Transtorno Dismórfico Corporal, em pacientes dermatológicos, e que os tratamentos cosméticos raramente melhoram seus sintomas, o treinamento dos profissionais para a investigação sistemática, diagnóstico e encaminhamento para tratamento psiquiátrico é fundamental.

Palavras-chave: Cirurgia plástica; Dermatologia; Imagem corporal; Prevalência; Transtorno obsessivocompulsivo; Transtornos somatoformes; Transtornos somatoformes/epidemiologia
\end{abstract}

\begin{abstract}
Cosmetic concerns are becoming increasingly common in view of the obsession with the perfect body and skin. Dermatologists are often seen to evaluate and treat these conditions. Therefore, it is important to acknowledge the existence of Body Dysmorphic Disorder, also known as dysmorphophobia. Despite being relatively common, this disorder has not been well researched. Sometimes causing impairment, the disease involves a distorted body image perception characterized by excessive preoccupation with a perceived defect. Most of the patients experience some degree of impairment in social or occupational functioning and, as a result of their obsessive concerns, they may develop compulsive behaviors. In severe cases, there is a risk of suicide. Most individuals do not acknowledge that their defect is minimal or nonexistent and seek out cosmetic treatments for a psychiatric disorder. The prevalence of this disorder among the general population ranges from 1 to $2 \%$ and in dermatological and cosmetic surgery patients, from 2.9 to $16 \%$. The training of professionals to systematically investigate, diagnose, and refer these patients to adequate psychiatric treatment is essential, considering the high prevalence of Body Dysmorphic Disorder in dermatological patients and the fact that cosmetic treatments rarely improve their condition.

Keywords: Body image; Dermatology; Obsessive-compulsive disorder; Prevalence; Somatoform disorders; Somatoform disorders/epidemiology; Surgery, plastic
\end{abstract}

\footnotetext{
Aprovado pelo Conselho Editorial e aceito para publicação em 21.09.2009.

* Trabalho realizado na Faculda de Medicina da USP (Deptos de Dermatologia e Psiquiatria)

Conflito de interesse: Nenhum / Conflict of interest: None

Suporte financeiro: Nenhum / Financial funding: None

Doutora em Ciências de Saúde pela Faculdade de Medicina da Universidade de São Paulo (FMUSP) - São Paulo (SP), Brasil

Mestre em Ciências de Saúde pela Faculdade de Medicina da Universidade de São Paulo (FMUSP) - São Paulo (SP), Brasil.

(C)2009 by Anais Brasileiros de Dermatologia
} 


\section{INTRODUÇÃO}

A insatisfação com a própria imagem parece ser comum $^{1}$ na população geral. No entanto, o nível de preocupação causado pela insatisfação com a imagem pode variar entre os indivíduos e atingir um grau em que estas preocupações causem interferência no seu funcionamento cotidiano. A insatisfação com a imagem corporal desempenha importante papel em um grande número de transtornos psiquiátricos, incluindo os transtornos alimentares, a fobia social, o transtorno de identidade de gênero, mas principalmente na condição psiquiátrica que é muito relevante para os dermatologistas: o Transtorno Dismórfico Corporal (TDC). A extrema insatisfação com a imagem corporal é o sintoma nuclear do TDC, e essa é a única categoria diagnóstica no "Diagnostic and Statistical Manual of Mental Disorders" (DSM) americano que diretamente se refere às queixas com a imagem corporal.

O TDC é relativamente comum, por vezes incapacitante, e envolve uma percepção distorcida da imagem corporal, caracterizada pela preocupação exagerada com uma anomalia imaginária na aparência ou com um mínimo defeito corporal presente. ${ }^{2}$

Apesar do importante aspecto social que envolve esse transtorno, foi pouco estudado até recentemente, e não reconhecido e diagnosticado por profissionais de saúde. ${ }^{3}$

\section{HISTÓRICO DO DIAGNÓSTICO DO TDC}

Em 1886, O TDC foi descrito por Enrico Morselli e denominado, inicialmente, de "dismorfofobia". $4 * *$

Janet (1903) descreveu a "l'obsession de bonte $d u$ corps" (obsessão com vergonha do corpo), Kraeplin (1909) o denominou como "dysmorpbopbobic syndrome", e os japoneses como "shubo-kyofu."

Antes da inclusão desse transtorno no "Diagnostic and Statistical Manual of Mental Disorders" (DSM), da Associação Norte-americana de Psiquiatria ou no "International Classification of Mental and Behavioural Disorders" (CID), da Organização Mundial de Saúde, há relatos de casos de pacientes, com sintomas consistentes com o TDC, na literatura de cirurgia cosmética e Dermatologia. Relatos dos anos 60 descrevem pacientes cirúrgicos, com mínimas deformidades e "insaciáveis"6, em relação à realização de cirurgias plásticas cosméticas. $\mathrm{Na}$ literatura dermatológica, encontramos descrições de pacientes cujo quadro clínico é denominado: dismorfofobia e "dermatological nondisease," síndrome dismórfica ou ainda hipocondria dermatológica. ${ }^{8}$ Utilizou-se o termo "escoriações psicogênicas", principalmente, na literatura dermatológica, para os pacientes que manipulavam frequentemente a pele. ${ }^{9}$ Nestes, os pacientes mostravam altos níveis de insatisfação com os resultados de tratamentos objetivamente aceitáveis, ou, a seguir, focavam sua preocupação, em outra área do corpo, ${ }^{9}$ uma vez que esses estudos não tinham critérios diagnósticos rigorosos. É possível que alguns, mas não todos os pacientes tivessem o TDC.

Apesar de haver sido descrito há mais de um século, o TDC não foi incluído, nos sistemas diagnósticos, até 1980, e sua classificação é, até hoje, discutida. ${ }^{10}$ Durante muito tempo, foi considerado sintoma de doenças psiquiátricas, tais como: a esquizofrenia, os transtornos de humor ou os transtornos de personalidade. ${ }^{11} \mathrm{Na}$ nosografia psiquiátrica norte-americana, o TDC é mencionado pela primeira vez no "Diagnostic and Statistical Manual of Mental Disorders (DSM)" -III (1980) ${ }^{12}$ como Transtorno Somatoforme Atípico e, no DSM III-R, (1987) ${ }^{13}$ com as variantes: não delirante e delirante.

Define-se Transtorno Somatoforme como "a presença de sintomas físicos que sugerem uma condição médica geral, mas que não são completamente explicados por uma condição médica geral, pelos efeitos diretos de uma substância ou por outro transtorno mental." $14 * * *$

No DSM-IV (1994) norte-americano, ${ }^{14}$ o TDC é classificado como "Body Dysmorphic Disorder" (BDD), uma das manifestações dos Transtornos Somatoformes.*

"Mais recentemente, o TDC tem sido reconhecido como síndrome específica, com possível categoria diagnóstica autônoma-, ou como mani-

\footnotetext{
* O sistema diagnóstico do DSM é multiaxial, no qual envolve cinco eixos, cada qual se refere a um domínio diferente de informações que possam contribuir para o diagnóstico clínico. São esses: Eixo I (Transtornos clínicos ou outras condições que possam ser foco de atenção clínica); Eixo II (Transtornos de personalidade); Eixo III (Condições Médicas Gerais); Eixo IV (Problemas psicossociais e relacionados ao ambiente) e Eixo $\mathrm{V}$ (diagnóstico do funcionamento global). ${ }^{23}$

** Do ponto de vista etimológico, o termo "dysmorphophobia" deriva do grego "dysmorphia”, significa "feiúra", particularmente em relação à face. No entanto, o transtorno não é caracterizado simplesmente pelo medo de ter um defeito físico, como o sufixo "phobia” pode sugerir. Além do mais, os pacientes manifestam o que pode ser descrito como uma polarização da atenção, com custo emocional, cujo foco é uma parte específica do corpo. O sujeito desenvolve repulsa por essa parte do corpo, o que causa interferência em diferentes graus, nas relações com os outros e no seu funcionamento social. ${ }^{14}$

*** Transtornos Somatoformes: ${ }^{24}$ hipocondria, transtornos de somatização, conversivos, dolorosos, dismórfico corporal, somatoformes indiferenciados, e de somatização sem outra especificação.
} 
festação do chamado espectro do Transtorno Obsessivo Compulsivo (TOC), que envolve transtornos que apresentam características psicopatológicas, de evolução clínica, padrão de comorbidades e respostas aos tratamentos semelhantes ao TOC ${ }^{15,16,17}$; ou ainda relacionado ao espectro das doenças afetivas. ${ }^{18}$ No entanto, as conclusões definitivas sobre essas relações ainda são limitadas pelo pouco conhecimento sobre a etiopatogenia do TDC."

\section{CRITÉRIOS DIAGNÓSTICOS PARA O TRANSTORNO DISMÓRFICO CORPORAL NO DSM-IV E NO CID-10}

Define-se o TDC como preocupação com "defeito" na aparência. O DSM-IV lista três critérios diagnósticos para TDC:

A) $\mathrm{O}$ indivíduo preocupa-se com um defeito imaginário na aparência e se uma mínima anomalia está presente, tem preocupação marcadamente excessiva com essa;

B) A preocupação deve causar estresse significativo ou prejuízo na vida social, ocupacional ou outras áreas do funcionamento;

C) Essas queixas não podem ser caracterizadas como outro transtorno mental, tais como a anorexia nervosa.

Na população de pacientes com queixas cosméticas, a aplicação dos critérios diagnósticos para o TDC pode ser um desafio.

O primeiro critério diagnóstico (A), se aplicado de forma independente, nas populações que buscam os profissionais da dermatologia cosmética, será positivo na maioria dos pacientes. Muitos pacientes desejam corrigir mínimos defeitos ou melhorar aspectos normais, e os profissionais são treinados para identificar e corrigir essas pequenas imperfeições na aparência. Além disso, nessa população, é normativo algum grau de insatisfação com a aparência para o qual o tratamento é desejado. ${ }^{19}$

O segundo critério diagnóstico (B), ou seja, o grau de insatisfação e prejuízo no funcionamento cotidiano pode ser o melhor indicador do TDC nesses pacientes. ${ }^{8}$ Por exemplo, se uma pessoa relata que suas queixas, em relação à aparência, interferem em sua habilidade em manter um trabalho, preenche critérios para o TDC. Em contraste, o diagnóstico do TDC é menos provável em um paciente que nega que suas preocupações tenham significante interferência no funcionamento social e ocupacional.

O critério $\mathrm{C}$ deve ser aplicado para excluir, principalmente, os transtornos alimentares. Por exemplo: um paciente com anorexia nervosa que tenha preocupações com a imagem corporal focadas - somente em "estar muito gordo" - deve receber apenas o diagnóstico de anorexia nervosa e não o do TDC. No entanto, os dois transtornos podem ocorrer simultaneamente. ${ }^{20}$ No CID-10,21 o critério para o diagnóstico do TDC exige que o paciente tenha procurado atenção médica para suas queixas com a aparência. Phillips ${ }^{20}$ aponta uma importante limitação desse critério, considerando que os pacientes com o TDC podem não ter procurado atendimento médico por diferentes razões (por exemplo: por não possuir seguro médico ou acesso ao tratamento, ou pela condição de isolamento social em que possam estar).

\section{CLÍNICA DO TRANSTORNO DISMÓRFICO CORPORAL}

Não há na literatura estudos epidemiológicos extensos ou completos sobre o TDC. No entanto, estudos retrospectivos ${ }^{22}$ e os dados do primeiro estudo prospectivo mostram importantes aspectos descritivos da clínica. ${ }^{23}$

A questão central do TDC é a crença de ser deformado, feio ou não ter atrativos em algum aspecto. $\mathrm{Na}$ realidade, o defeito na aparência é mínimo ou inexistente. Essa distorção cognitiva torna-se uma obsessão, e os pensamentos causam estresse e são difíceis de resistir e controlar, ${ }^{24}$ ocupando grande parte do dia. ${ }^{25} \mathrm{Na}$ realidade, o "defeito" freqüentemente é imperceptível a um interlocutor que esteja a uma distância normal de conversação. São percebidos pelo paciente como devastadores, causando ansiedade e estresse, fazendo com que busquem constantemente e de modo irracional a perfeição e simetria na sua aparência. ${ }^{26}$ Para diferenciar o TDC das queixas normais com a aparência, que são comuns na população geral, a preocupação deve causar estresse significativo ou prejuízo no funcionamento cotidiano. ${ }^{25}$

É mais comum que essas preocupações envolvam áreas da face (pele, cabelos e nariz), embora qualquer parte do corpo possa ser foco de preocupação. Apresentam com freqüência queixas relacionadas à acne, rugas e linhas de expressão; alterações na cor (p. ex. acreditam que a pele é muito vermelha ou muito branca) ou textura da pele; cicatrizes e estrias, "marcas" como nevus nevocelulares, cabelos finos ou excesso de pêlos no corpo; e queixas relativas ao tamanho e forma do nariz. ${ }^{7}$ Além da face, os homens têm como maior foco, preocupações com os genitais, peso, cabelos e formas do corpo, enquanto as mulheres mais tipicamente relatam preocupações com o peso, os quadris, as pernas e as mamas. ${ }^{27} \mathrm{Em}$ média, pessoas com o TDC relatam preocupações com cinco a sete partes do corpo durante o curso do transtorno.$^{28}$

Os pacientes relatam incapacidade para interromper os pensamentos sobre sua percepção do defeito, sendo difícil controlar tal obsessão, que pode tornar-se mais intensa em situações nas quais o indivíduo sente-se pressionado pela expectativa de ser 
observado, como em situações sociais. ${ }^{29}$ Tentando reduzir o estresse causado pelas suas crenças, os pacientes utilizam estratégias que geralmente tem pouco ou nenhum sucesso.

Os comportamentos mais comuns são: a reafirmação constante da aparência pela checagem no espelho ou em superfícies reflexivas (p. ex.: vidros) para se certificar que está normal ou "aceitável"; a camuflagem do "defeito" com os cabelos, maquiagem, óculos escuros ou roupas; controle da posição do corpo para evitar que o "defeito" seja observado; cuidados pessoais excessivos ("grooming behaviours") em salões de beleza, com mudanças de cor e corte de cabelos; ou utilizando maquiagens, produtos cosmecêuticos e tratamentos dermatológicos em excesso (filtros solares, ácido retinóico). Os comportamentos são variáveis e ilimitados: "bronzeamento artificial"; ${ }^{30}$ dietas freqüentes; exercícios excessivos e uso de esteróides anabolizantes caracterizando a "Dismorfia Muscular"; ${ }^{31}$ hábito de tocar ou medir partes do corpo comparando sua aparência com a de pessoas famosas; comprar em excesso produtos de beleza ou roupas; ler sobre a aparência física e o corpo. ${ }^{32}$

Alguns comportamentos tais como a manipulação da pele ou dos cabelos parece ser importante no TDC. A maioria das pessoas apresenta esses comportamentos em extensão limitada, várias vezes na vida. ${ }^{33}$ Esses comportamentos são complexos e tornam-se patológicos (escoriações patológicas ou tricotilomania) dependendo do seu foco, da duração e extensão, dos problemas resultantes, bem como das razões e emoções associadas. As escoriações patológicas ("skin picking") e a tricotilomania são recorrentes e podem resultar em danos consideráveis à pele e aos cabelos.

As "escoriações patológicas" ("skin picking") são reconhecidas há muitos anos na Dermatologia, chamadas de "dermatotilexomania" ou "escoriações neuróticas". ' Os pacientes relatam que esse comportamento é compulsivo e irresistível e muitas vezes podem piorar uma dermatose (p. ex. acne leve).$^{33}$ As "escoriações patológicas" podem ocorrer como sintoma de vários transtornos psiquiátricos $\mathrm{e}$, em estudo de pacientes com o TDC foi relatado em $27 \%$ dos pacientes. ${ }^{33}$ Embora esse comportamento seja considerado de "auto-injúria" os pacientes com o TDC não têm a intenção de se machucar. Seu desejo é tentar melhorar a aparência da pele. Podem utilizar alfinetes, facas, clipes e lâminas, causando muitas vezes lesões notáveis com infecções secundárias ou cicatrizes profundas e mais raramente lesando vasos importantes como a artéria carótida. ${ }^{20}$ Se na avaliação do paciente ficar claro que sua motivação é a queixa com a aparência e a melhora do "defeito", então é mais provável o diagnóstico do TDC. ${ }^{25}$
Da mesma forma, preocupações com os cabelos também são comuns, fazendo com que os pacientes procurem tratamento dermatológico. As queixas mais freqüentes são de queda de cabelos e medo de ficar calvo (especialmente homens). Muitos evidenciam ter poucos cabelos, mas geralmente os cabelos estão normais. Podem usar bonés, prendedores de cabelos, lenços e utilizar tônicos capilares, finasterida e minoxidil. Se a queixa for de excesso de pêlos podem utilizar lâminas, ceras depilatórias ou pinças para removê-los. Como nas escoriações patológicas a tentativa de remoção dos pêlos pode levar a infecção e cicatrizes. Também é importante diferenciar da tricotilomania: os pacientes com o TDC removem os pêlos para melhorar sua aparência; enquanto na tricotilomania, a remoção dos pêlos não é motivada por crenças ou pensamentos específicos. ${ }^{25}$

A maioria dos pacientes com o TDC tem prejuízos na vida social, acadêmica ou ocupacional como resultado de suas preocupações e qualidade de vida ruim. A gravidade do TDC também é variável entre os pacientes, havendo aqueles que levam uma vida aparentemente normal apesar do sofrimento e dos prejuízos cotidianos que têm. Nos casos mais graves o paciente evita situações de contato com outras pessoas, não consegue trabalhar ou ter relacionamentos sociais e afetivos devido à preocupação de parecer "feio" ou ao medo de que outras pessoas estejam fazendo comentários depreciativos em relação ao seu "defeito". ${ }^{35}$ Mostram-se agressivos quando são impedidos de realizar seus comportamentos ou em situações estressantes. ${ }^{36}$ Nos casos de gravidade extrema, há risco de suicídio. ${ }^{4,37}$

Os níveis de crítica sobre as preocupações com a aparência podem variar durante o curso da enfermidade: há pacientes que apresentam juízo crítico bom ou razoável, percebendo que suas preocupações são um tanto exageradas e pacientes que não reconhecem que suas preocupações são excessivas. A classificação em separado das formas "delirante" e não delirante é contrária á experiência clínica e ás crescentes evidências na literatura, que mostram que o TDC é um transtorno único e a forma chamada "delirante", apenas mais grave. Além disso, pacientes com capacidade crítica ("insight") prejudicado no início do tratamento podem apresentar progressiva melhora nesse aspecto.

Há poucos estudos prospectivos avaliando os tratamentos dermatológicos e de cirurgia plástica nesses pacientes. Entretanto, com base em observações clínicas, os pacientes com o TDC, muitas vezes sem esse diagnóstico formal, são descritos pela busca freqüente de tratamentos cosméticos tais como terapias com laser, dermabrasão, transplante de cabelos e cirurgias plásticas, mesmo que esses tratamentos não tenham indicação médica. ${ }^{22}$ Outro aspecto clínico 
importante é a vergonha que sentem dos seus sintomas e, consequentemente de relatá-los ao profissional. Acreditando que seu problema seja cosmético, procuram tratamento em especialistas nessa área.

\section{PREVALÊNCIA DO TRANSTORNO DISMÓRFICO CORPORAL}

Transtorno Dismórfico Corporal na população geral

A prevalência do TDC, na população geral, ainda não é bem estabelecida. No entanto, estima-se que afete, aproximadamente, de 1 a $2 \%$ da população geral. ${ }^{38}$ Estudos mais antigos relataram prevalência de $0,7 \%$, na população geral, ${ }^{19}$ e outro mostrou de 1 a $3 \% .39$ Os mais recentes, avaliando amostras maiores, indicam prevalência de $1,7 \%$ na Alemanha e nos Estados Unidos de 2,4\%.40,41

Nas populações de estudantes, as prevalências do TDC variam de 2,5 a $28 \% .^{8,19,42}$ Um estudo demonstrou que não houve diferenças significativas entre as amostras de estudantes norte-americanos (4\%) e alemães $(5,3 \%),{ }^{42}$ utilizando-se de amostras e métodos similares.

\section{Transtorno Dismórfico Corporal em populações de dermatologia e cirurgia plástica}

Estudos empíricos sugerem que a prevalência do TDC nesses pacientes parece ser maior do que na população geral. Um estudo norte-americano, de $1998,{ }^{8}$ descreve que a prevalência do TDC varia entre 7 e $8 \%$, e dois estudos europeus mostraram prevalências de $6,3 \%$ e $9,1 \%, 43,44$ nos pacientes de cirurgia plástica cosmética.

No entanto, as prevalências, na literatura, variaram de 2,9 a 53,6\%,19 mas, em estudos que não utilizaram a mesma metodologia, alguns com pequenas amostras, vieses de seleção e uso de entrevistas nãoestruturadas.

Nos pacientes que procuram consultas clínicas na Dermatologia, a prevalência do TDC parece ser ligeiramente maior do que em populações de cirurgia cosmética.2,19,20 Segundo Phillips et al.,2 o dermatologista pode ser o profissional que mais frequentemente é consultado por esses pacientes. Em estudo de prevalência, em população dermatológica clínica, $14,4 \%$ dos pacientes tiveram critérios diagnósticos para o TDC e a maioria desses procurou tratamento para acne. ${ }^{2}$

O TDC parece ser mais prevalente nessas populações, mostrando a importância do conhecimento desse transtorno e de seus aspectos clínicos para estes profissionais.

\section{Transtorno Dismórfico Corporal em populações de pacientes psiquiátricos}

Um estudo de $1998^{45}$ mostrou prevalência de $3,2 \%$ do TDC entre os pacientes de clínica psiquiátrica ambulatorial. Fontenelle et al. ${ }^{46}$ encontraram $12 \%$ de prevalência em pacientes psiquiátricos ambulatoriais no Brasil. Apenas dois estudos investigaram o TDC em pacientes psiquiátricos internados por outros diagnósticos. Esses mostraram que de 13 a 16\% dos pacientes tinham critérios diagnósticos para o TDC no momento da entrevista ou tiveram em algum momento de suas vidas. ${ }^{47}$ Nenhum paciente havia recebido o diagnóstico do TDC durante sua hospitalização, mesmo que alguns pacientes considerassem seus sintomas do TDC como o "seu maior problema". Todos os pacientes disseram que não revelariam seus sintomas espontaneamente ao seu médico por sentirem vergonha.

\section{Transtorno Dismórfico Corporal em outras populações médicas}

Em outras populações médicas, os estudos são mais escassos. Na clínica médica geral, $4 \%$ dos pacientes tiveram critérios para o $\mathrm{TDC}^{20} \mathrm{e}$, em um estudo de população de pacientes ortodônticos, $7,5 \% .{ }^{48}$ Não existem estudos de pacientes, nas populações que procuram tratamento cosmético com profissionais paramédicos, mas alguns sugerem que pacientes com o TDC, com frequência, buscam esses profissionais. ${ }^{28,49} \mathrm{O}$ estudo de Kittler et al. ${ }^{50}$ apontou que pacientes obesos também podem apresentar o TDC e, com o desenvolvimento da cirurgia bariátrica, nos últimos anos, tem havido grande procura por tratamentos cosméticos para melhorar as consequências da redução drástica de peso. No entanto, pouco se sabe sobre os aspectos psicológicos desses pacientes. ${ }^{1}$

\section{ASPECTOS DEMOGRÁFICOS \\ Idade}

Inicia-se em geral na adolescência, podendo ocorrer também na infância. Em média os pacientes sofrem por até onze anos antes que procurem tratamento específico. ${ }^{20} \mathrm{O}$ extenso estudo de Phillips et al. ${ }^{23}$ mostrou que, nos pacientes com o TDC, a média de idade de desenvolvimento do transtorno foi de 16,4 anos ( \pm 7 anos), embora a insatisfação com a aparência tenha se manifestado entre 12,9 anos $( \pm 5,8$ anos). Parece haver um segundo pico de incidência ocorrendo após a menopausa. ${ }^{10}$

\section{Diferenças de gênero}

Embora não seja definitivo, parece não haver diferenças entre os gêneros. ${ }^{19}$ Alguns estudos mostraram maior freqüência nas mulheres ${ }^{19,24}$ outros em homens. ${ }^{9,51,52}$ Nos estudos de Rief et al..$^{41}$ e Koran et 
al., ${ }^{40}$ que observaram as maiores amostras populacionais, o TDC foi ligeiramente mais prevalente entre as mulheres (1,9: 1,4\% e 2,5: 2,2\%, respectivamente). No entanto, homens e mulheres parecem similares quanto à maior parte dos aspectos clínicos e demográficos. ${ }^{23,27}$

No estudo de Phillips et $a .^{27}$ os homens eram mais velhos, solteiros e preocupados com os genitais, com a massa muscular e com os cabelos; enquanto as mulheres apresentaram maior preocupação com as mamas, com as coxas e com as pernas, e também uma maior tendência a camuflar o suposto defeito.

\section{ETIOLOGIA}

\section{Genética do Transtorno Dismórfico Corporal}

Os fatores genéticos parecem desempenhar importante papel na etiologia do TDC, como evidenciado pelos padrões de hereditariedade observados. $^{39}$ Dos indivíduos com o TDC, 8\% têm algum membro da família com esse diagnóstico durante a vida, o que representa de 4 a 8 vezes a prevalência na população geral. ${ }^{39}$

O TDC compartilha a hereditariedade com o TOC, como mostrou um estudo familiar no qual $7 \%$ dos pacientes com o TDC tinham algum familiar de primeiro grau com o TOC. ${ }^{53}$

\section{Fatores neurobiológicos}

No desenvolvimento do TDC, funções anormais da serotonina e da dopamina podem estar envolvidas, como foi evidenciado pela boa resposta desses pacientes a medicações que alteram os níveis desses neurotransmissores. ${ }^{19}$ Relatos de casos sugerem que o desenvolvimento do TDC possa ter como gatilho, patologias clínicas inflamatórias, que interferem na síntese de serotonina; sofrer exacerbação dos sintomas, após infecção estreptocóccica, ${ }^{54}$ ou ainda, surgir, após lesão na região do lobo frontotemporal. 55

\section{Fisiopatologia - modelo teórico}

Embora a fisiopatologia do TDC ainda seja desconhecida, Feusner et al., 53 observando casos clínicos com lesões cerebrais e estudos de neuroimagem com ativação cerebral que mostraram padrões de percepção visual, imagem corporal distorcida e processamento emocional, propuseram um modelo para compreensão das disfunções neuroanatômicas, possivelmente, envolvidas nos sintomas do TDC. Esse modelo propõe que ocorra uma combinação de disfunções, no circuito fronto-estriatal, nos balanços entre os hemisférios cerebrais e maiores graus de respostas, na amígdala e na ínsula, mediando os sintomas e déficits neuropsicológicos no TDC.

\section{Fatores psicológicos}

Pelo menos duas teorias psicológicas se propuseram a esclarecer as causas do TDC: a teoria psicanalítica e a cognitivo-comportamental. A teoria psicanalítica sugere que o TDC emerge do deslocamento inconsciente de conflitos sexuais ou emocionais, de sentimentos de inferioridade, culpa ou distorção da imagem corporal de alguma parte do corpo. Explicações, a partir de um ponto de vista cognitivocomportamental, sugerem que o TDC manifesta-se de uma interação entre fatores comportamentais, cognitivos e emocionais. ${ }^{24}$ Os fatores cognitivos que parecem ser instrumentais no desenvolvimento $e$ manutenção do TDC incluem atitudes não realistas sobre a imagem corporal relacionadas à perfeição e simetria; atenção seletiva aos defeitos percebidos e aumento do monitoramento da presença de defeitos na aparência, além da interpretação errônea das expressões faciais dos outros como sendo, por exemplo, de crítica ou de raiva. ${ }^{56}$ As pessoas com o TDC tendem a achar que sua aparência é bem menos atrativa do que acreditam ser a ideal. ${ }^{24}$

\section{COMORBIDADES}

A maioria dos pacientes com o TDC tem pelo menos um transtorno mental em comorbidade. As comorbidades são muito comuns e, no maior estudo em pacientes com o TDC, Gustand e Phillips ${ }^{57}$ mostraram que havia critérios diagnósticos para mais de duas outras comorbidades, ao longo da vida (Eixo I / DSMIV)*. Um maior número de comorbidades é associado a maior morbidade e prejuízo funcional.57 As comorbidades mais freqüentes parecem ser os transtornos de humor e ansiedade, os transtornos do espectro obsessivo-compulsivo, os transtornos alimentares, os transtornos do uso de substâncias ${ }^{57}$ e os transtornos de personalidade. ${ }^{58}$

\section{Transtornos de Humor}

O Transtorno Depressivo Maior (TDM) parece ser a comorbidade mais freqüente. Mais de $75 \%$ dos pacientes com o TDC teve, ao menos, um episódio, ao longo da vida e, pelo menos a metade dos pacientes tem critérios para o diagnóstico de Episódio Depressivo Maior Atual. ${ }^{19}$

Alguns autores sugeriram que o TDC seria um sintoma de depressão ou transtorno relacionado ao espectro dos transtornos afetivos, uma vez que ambos se caracterizam por baixa autoestima, sentimentos de rejeição e desvalia e labilidade emocional. No entanto, o TDC difere da depressão, principalmente, pela presença de pensamentos obsessivos e comportamentos compulsivos. Os pacientes com depressão, tipicamente, se preocupam menos com a aparência e não focam em aspecto específico dessa. ${ }^{10}$ 
No entanto, como no TDM, os pacientes com TDC também estão mais propensos à ideação e tentativas de suicídio. Em estudo recente, em que foram avaliados 200 pacientes com o TDC, Phillips et al.59 relatam frequência de $78 \%$ de idealização suicida e $27,5 \%$ de tentativas de suicídio, sendo o TDC a principal motivação referida pelos pacientes.

\section{Transtornos de Ansiedade}

Nos pacientes com o TDC, os Transtornos de Ansiedade são freqüentes. No maior estudo de comorbidades, em pacientes com o TDC, os Transtornos de Ansiedade foram relatados em $60 \%$ dos pacientes, ao longo da vida ${ }^{15}$ e a Fobia Social teve prevalência de $38 \%$, tendendo a preceder o TDC. 58 Semelhante ao TDC, a Fobia Social é caracterizada por ansiedade social e comportamentos de evitação. No entanto, na Fobia Social, não há foco específico em um aspecto particular da face ou do corpo. ${ }^{60}$ Phillips et al. ${ }^{61}$ sugerem que a Fobia Social se desenvolve secundariamente nos pacientes com o TDC.

\section{Transtornos do Espectro Obsessivo-Compulsivo (Espectro TOC)}

O TDC é, há muito tempo, relacionado ao Transtorno Obsessivo-Compulsivo (TOC) e conceituado como parte dos Transtornos do Espectro Obsessivo-Compulsivo. 5 Portanto, fazem-se pertinentes algumas considerações sobre as relações entre $o$ TDC e o TOC.

O TOC é um transtorno crônico, que causa prejuízo funcional, caracterizado por obsessões recorrentes e/ou comportamentos compulsivos que são executados para aliviar o incômodo causado pelos pensamentos invasivos. As obsessões mais comuns envolvem as dimensões de contaminação, imagens sexuais, agressão, dúvidas, ordem e simetria. As obsessões são egodistônicas, ou seja, os pacientes com o TOC descrevem seus pensamentos obsessivos como "estrangeiros" e não derivados de sua mente, impostos extrinsecamente. As dimensões de compulsões mais habituais envolvem contaminação, lavagem, checagem, ordem e arranjo, sendo que tais obsessões e compulsões consomem tempo e contribuem para prejuízo social e estresse significativo, no cotidiano desses pacientes. É comum também observarmos os comportamentos de evitação, relacionados à tentativa de se evadir de situações e coisas (p. ex. objetos) que possam servir de gatilho para os sintomas. ${ }^{9}$

O TOC atualmente é classificado como Transtorno de Ansiedade (DSM-IV), mas sua reclassificação como parte dos Transtornos do Espectro Obsessivo-Compulsivo é motivo de debate e vem ganhando proeminência. ${ }^{9}$
Hollander et al. ${ }^{9}$ ressaltam que os estudos de comorbidades, familiares e neurológicos têm evidenciado as similaridades na sintomatologia, curso do transtorno, população de pacientes e neurocircuitaria do TOC e dos Transtornos do Espectro TOC, apontando também para uma revisão crítica das relações entre o TOC e os Transtornos de Ansiedade. Segundo esses autores, a razão principal para classificar o TOC como Transtorno de Ansiedade é ser a ansiedade o fator central no TOC. As obsessões associadas ao TOC contribuem para a crescente ansiedade e os comportamentos compulsivos são freqüentemente executados para tentar reduzir essa ansiedade. No entanto, a ansiedade como sintoma também é observada em outros transtornos psiquiátricos entre esses a depressão e a esquizofrenia. ${ }^{9}$ O TOC é um transtorno clinicamente heterogêneo (resultante de possíveis expressões fenotípicas diferentes) e, de um ponto de vista de suas dimensões, pode-se observar sobreposição de sintomas em diferentes transtornos do Espectro TOC.

Hollander et al. ${ }^{9}$ propuseram a subdivisão dos Transtornos do Espectro Obsessivo-Compulsivo em três subgrupos:

1) Imagem corporal e transtornos somáticos, caracterizados por obsessóes com o corpo que incluem hipocondríase, compulsão alimentar, anorexia nervosa, o TDC e transtorno de despersonalização;

2) Transtornos de controle de impulsos, tais como: transtornos sexuais que envolvem obsessões, compulsões ou parafilias, jogo patológico, tricotilomania, comprar compulsivo, abuso de internet e escoriações patológicas ("skin picking"). De modo similar ao TOC, os pacientes experimentam uma sensação de tensão e emergência, associadas a esses comportamentos, mas ao contrário do TOC, estas atitudes trazem um curto período de prazer (que não está presente no TOC);

3) Transtornos neurobiólogicos com comportamentos repetitivos, a saber: a Síndrome de Tourette, Corea de Sideham e o autismo. Nesses transtornos, as compulsões são comportamentos motores repetitivos e, usualmente, sem obsessões;

Há muito tempo são reconhecidas as similaridades entre o TDC e o TOC. Quando Morselli identificou os pacientes com "body dysmorphic syndrome" (dysmorphophobia) há mais de um século, notou as manifestações obsessivas e os comportamentos compulsivos que caracterizam esses pacientes. ${ }^{62}$ No entanto, parece haver consenso entre os autores de que o TDC, embora relacionado ao TOC, não seja apenas variante clínica deste, apresentando diferenças significativas. ${ }^{10,5,11,62}$ Há sobreposição entre o TDC e o TOC, quanto à idade de aparecimento, distribuição entre os sexos, curso crônico, história familiar de 
transtornos psiquiátricos e resposta ao tratamento.

Frare et al. ${ }^{62}$ ressaltam que, do ponto de vista clínico, as principais similaridades entre o TDC e o TOC evidenciam-se pelas características das preocupações, por serem obsessivas e persistentes; com pensamentos que causam incômodos, produzem ansiedade e são difíceis de resistir e controlar. Mas no TDC estão mais presentes sentimentos de vergonha, humilhação, baixa autoestima, rejeição e pensamentos de referência. Os comportamentos repetitivos lembram as compulsões do TOC.

As características de alguns sintomas são similares, tais como as preocupações com a simetria (na posição de objetos ou do próprio corpo), a procura da perfeição, necessidade de controlar o ambiente, de reasseguramento frequente, além dos comportamentos de checagem. No entanto, os focos das preocupações são diferentes entre os transtornos: no TDC, a aparência física; e no TOC, medo de contaminação ou outros medos. Também verificamos diferenças com relação aos comportamentos de checagem (por exemplo: fixação no espelho): no TDC apresentam padrão mais complexo (ligado à segurança) do que no modelo proposto para o TOC (ligado à redução da ansiedade). Esses comportamentos de checagem, de fato, parecem ser menos eficazes em reduzir a ansiedade no TDC do que no TOC.

Os mesmos autores ${ }^{62}$ apontam para a principal diferença entre o TDC e o TOC: o nível de juízo crítico ("insight") sobre os sintomas. As preocupações no TDC em geral são vivenciadas de modo mais natural ou egossintônico (portanto menos intrusivas) e os pacientes as aceitam com algum grau de convicção e cedem a elas sem tanta resistência.

Enquanto no TOC geralmente as preocupações são mais egodistônicas e, portanto, vivenciadas como mais intrusivas (irracionais, exageradas), no TDC muitos pacientes estão convencidos de que a sua visão sobre o suposto defeito é acurada e não distorcida. Além disso, vários deles também apresentam idéias de autorreferência, acreditando que as outras pessoas estão frequentemente olhando para seu problema cosmético. Ao contrário do observado no TOC, muitos pacientes estão completamente convencidos de que sua visão do suposto defeito é acurada e não distorcida e a maioria dos pacientes também tem idéias ou delírios de referência. Um estudo clínico mostrou que nos pacientes que apresentam TOC e TDC associado, o nível de juízo crítico ("insight") foi significantemente mais prejudicado em relação ás preocupações com as dismorfias corporais do que em relação aos sintomas do TOC. ${ }^{8}$ Além da pior capacidade de crítica no TDC os pacientes também apresentam maior predisposição à ideação e tentativas de suicídio do que no TOC. ${ }^{63}$ Entretanto, apesar dessas evidên- cias, há poucas investigações sobre as similaridades e as diferenças entre o TDC e o TOC, bem como sobre as repercussões clínicas da associação entre esses transtornos.

O TDC tem alta comorbidade com os Transtornos do Espectro TOC, variando a freqüência de associação ao TOC de $30^{57}$ a $78 \% 15$ ao longo da vida. O TDC também apresenta aspectos de superposição com outros Transtornos do Espectro TOC, tais como tricotilomania ${ }^{63}$ e escoriações patológicas ("skin picking"). ${ }^{64}$

\section{Uso e Abuso de Substâncias}

Vários estudos relataram taxas elevadas de abuso de substâncias em pacientes com o TDC. 10,57 Grant e Phillips ${ }^{26}$ relataram abuso em 48,9\% ( $n=86$ ) e dependência em 35,8\%, particularmente ao álcool (29\%). Em 68\% desses pacientes o TDC foi o principal elemento causal relacionado ao abuso de substâncias.

\section{Transtornos Alimentares}

Os Transtornos Alimentares parecem, relativamente, comuns, em indivíduos com o TDC. Alguns autores sugeriram que a distorção na imagem corporal pode ser o principal aspecto da patologia, tanto no TDC como nos Transtornos Alimentares. Ruffolo et. al.32 encontraram incidência de $32,5 \%$ de Transtornos Alimentares (Anorexia Nervosa, Bulimia Nervosa e outros Transtornos Alimentares não especificados), ao longo da vida, nos pacientes com o TDC. Comparando os grupos com e sem Transtornos Alimentares, esses autores mostraram que os pacientes comorbidos eram, preferencialmente, mulheres e com insatisfação e distúrbios mais significativos da imagem corporal do que os pacientes apenas com o TDC.

\section{Transtornos de Personalidade}

Parece haver uma alta prevalência de Transtornos de Personalidade entre pacientes com o TDC. Em um estudo das comorbidades do Eixo II, $57 \%$ dos pacientes com o TDC apresentaram critérios diagnósticos para, pelo menos, um Transtorno de Personalidade, mais habitualmente, o tipo evitador. ${ }^{19}$ Personalidades paranoide, obsessivo-compulsiva e dependente também podem ocorrer com o TDC. ${ }^{19}$

\section{TRATAMENTO}

\section{Tratamento Farmacológico}

O TDC tem melhor resposta com os inibidores seletivos da recaptação da serotonina (ISRS), mas também responde aos antidepressivos tricíclicos. Um estudo retrospectivo de Hollander et al. apud Pavan et al. ${ }^{10}$ mostrou melhor remissão dos sintomas do TDC, quando se utilizava fluoxetina, fluvoxamina ou 
clomipramina. Estudo duplo-cego conduzido por Phillips, ${ }^{65}$ comparando fluoxetina e placebo, confirmou sua eficácia no tratamento do TDC. Em outro estudo, esses autores sugerem que há necessidade de observação de protocolos para adequação de doses terapêuticas, ${ }^{64}$ uma vez que, em apenas $34,4 \%$ dos pacientes, as doses foram consideradas minimamente adequadas para o TDC. Phillips ${ }^{66}$ mostrou boa eficácia terapêutica com o citalopram, mas a suspensão da medicação levou à recaída dos sintomas em 83,3\% dos pacientes, em média, em 38 semanas. Esses estudos mostraram que a presença de características delirantes não parece ser fator preditivo de má resposta ao tratamento com os ISRS. Os pacientes respondem ao tratamento com redução das preocupações, diminuição do incômodo, e diminuição dos comportamentos rituais, além de significante melhora no funcionamento social e ocupacional. O defeito geralmente é notado (é raro que não seja mais percebido), mas usualmente causa menos incômodo. ${ }^{10}$

\section{Tratamento Psicoterapêutico}

A eficácia da terapia cognitivo-comportamental (TCC) para o TDC vem sendo comprovada com estudos randomizados e ensaios clínicos controlados. ${ }^{19}$ Envolve a identificação e modificação das cognições e comportamentos "problema" relacionados à aparência. As estratégias, utilizadas na TCC, incluem o automonitoramento dos pensamentos e comportamentos relacionados à aparência (por exemplo: controle do tempo gasto na checagem no espelho); técnicas cognitivas (por exemplo: desafiando os pensamentos sobre sua aparência); e exercícios comportamentais (por exemplo: exposição do paciente a situações de medo e prevenindo o engajamento em comportamentos compulsivos). ${ }^{67,68}$

Ainda não há estudos com avaliação rigorosa da combinação de tratamentos psicológicos e farmacológicos para o TDC.

\section{CONCLUSÕES}

Segundo Phillips e Dufresne, ${ }^{69}$ nos últimos anos, o TDC deixou de ser um transtorno psiquiátrico negligenciado e está sendo mais bem reconhecido e compreendido. Trata-se de um transtorno grave, relativamente comum que, frequentemente, apresenta-se aos profissionais de saúde mental e também a médicos não psiquiatras. Muitos pacientes sentem vergonha dos seus sintomas e não os relatam, mesmo que desejem que seu médico saiba de suas queixas com a aparência. ${ }^{3}$ Em média, o paciente procura tratamento específico, após onze anos do aparecimento dos primeiros sintomas. ${ }^{3}$

Provavelmente o TDC esteja sendo subestimado, pois poucos psiquiatras ou especialistas que fazem um primeiro contato com os pacientes com o TDC reconhecem essa condição. ${ }^{10} \mathrm{Em}$ contraste, observa-se que os pacientes com o TDC consultam dermatologistas, cirurgiões plásticos e outros profissionais, a fim de modificar sua aparência, ${ }^{3}$ mostrando-se insatisfeitos com os resultados e solicitando novas intervenções para a mesma queixa ou para uma área do corpo diferente, para a qual transferiram o foco das atenções. É da maior importância que esses profissionais conheçam os sintomas do TDC e investiguem especificamente esses sintomas. Os tratamentos cosméticos parecem ineficazes no TDC e podem oferecer riscos aos médicos que os executam, uma vez que os pacientes podem tornar-se agressivos e violentos com seus médicos. Além do mais, esses têm maiores taxas de ideação suicida e/ou tentativas de suicídio, incluindo efetivas.

Também parece ser fundamental que os dermatologistas sejam treinados para identificar pacientes subclínicos ou com queixas que sinalizem a presença do TDC, pois estão em uma posição estratégica para reconhecer os sintomas dos pacientes e conduzi-los ao tratamento apropriado. $\mathrm{O}$ encaminhamento desses pacientes para o psiquiatra é difícil, posto que, por ter o nível de juízo crítico prejudicado, eles não reconhecem que sua visão do defeito é distorcida e que as queixas com a aparência são devidas a um transtorno psiquiátrico. 


\section{REFERÊNCIAS}

1. Sarwer DB, Crerand CE. Body dysmorphic disorder and appearance enhancing medical treatments. Body Image. 2008;5:50-8.

2. Phillips KA, Dufresne RG Jr, Wilkel CS, Vittorio CC. Rate of body dysmorphic disorder in dermatology patients. J Am Acad Dermatol. 2000;42:436-41.

3. Phillips KA. The broken mirror: understanding and treating Body Dysmorphic Disorder. New York: Oxford University Press, 1996.

4. Sarwer DB, Crerand CE, Didie ER. Body dysmorphic disorder in cosmetic surgery patients. Facial Plast Surg. 2003; 19:7-18.

5. Phillips KA, Kaye WH. The relationship of body dysmorphic disorder and eating disorders to obsessive-compulsive disorder. CNS Spectr. 2007;12:347-58.

6. Edgerton MT, Jacobson WE, Meyer E. Surgicalpsychiatric study of patients seeking plastic (cosmetic) surgery: ninety-eight consecutive patients with minimal deformity. Br J Plast Surg. 1960;13:136-45.

7. Cotterill JA. Body dysmorphic disorder. Dermatol Clin. 1996; 14:457-63.

8. Sarwer DB, Wadden TA, Pertschuk MJ, Whitaker LA. Body image dissatisfaction and body dysmorphic disorder in 100 cosmetic surgery patients. Plast Reconstr Surg. 1998;101:1644-9.

9. Hollander E, Neville D, Frenkel M, Josephson S, Liebowitz MR. Body dysmorphic disorder. Diagnostic issues and related disorders. Psychosomatics. 1992;33:156-65.

10. Pavan C, Simonato P, Marini M, Mazzoleni F, Pavan L, Vindigni V. Psychopathologic aspects of body dysmorphic disorder: a literature review. Aesthetic Plast Surg. 2008;32:473-84

11. Perugi G, Akiskal HS, Giannotti D, Frare F, Di Vaio S, Cassano GB. Gender-related differences in body dysmorphic disorder (dysmorphophobia). J Nerv Ment Dis. 1997; 185:578-82.

12. American Psychiatric Association. Diagnostic and Statistical Manual of Mental Disorders. 3rd ed. Washington, D.C.: American Psychiatric Association, 1980.

13. American Psychiatric Association. Diagnostic and Statistical Manual of Mental Disorders. 3rd ed. revised. 1987.

14. American Psychiatric Association. Diagnostic and statistical manual of mental disorders (DSM-IV). 4th ed. Washington (DC): American Psychiatric Association, 1994.

15. Simeon D, Hollander E, Stein DJ, Cohen L, Aronowitz B. Body dysmorphic disorder in the DSM-IV field trial for obsessive-compulsive disorder. Am J Psychiatry. 1995;152:1207-9.

16. Hounie AG, Pauls DL, Mercadante MT, Rosario-Campos MC, Shavitt RG, de Mathis MA, et al. Obsessivecompulsive spectrum disorders in rheumatic fever with and without Sydenham's chorea. J Clin Psychiatry. 2004;65:994-9.

17. Castle DJ, Phillips KA. Obsessive-compulsive spectrum of disorders: a defensible construct? Aust $\mathrm{N} \mathrm{Z} \mathrm{J}$ Psychiatry. 2006; 40:114-20.

18. Phillips KA, McElroy SL, Hudson JI, Pope HG Jr. Body dysmorphic disorder: an obsessive-compulsive spectrum disorder, a form of affective spectrum disorder, or both? J Clin Psychiatry. 1995;56 Suppl 4:41-51; discussion 52.

19. Crerand CE, Franklin ME, Sarwer DB. Body dysmorphic disorder and cosmetic surgery. Plast Reconstr Surg. 2006;118:167e-80e.

20. Phillips KA. The Presentation of Body Dysmorphic Disorder in Medical Settings. Prim Psychiatry. 2006;13:51-9.

21. World Health Organization. International Classification of Mental and Behavioural Disorders. Tenth revision (ICD 10) Geneva: WHO, 1992.

22. Castle DJ, Rossell S, Kyrios M. Body dysmorphic disorder. Psychiatr Clin North Am. 2006;29:521-38.

23. Phillips KA, Menard W, Fay C, Weisberg R. Demographic characteristics, phenomenology, comorbidity, and family history in 200 individuals with body dysmorphic disorder. Psychosomatics. 2005;46:317-25.

24. Veale D. Advances in a cognitive behavioural model of body dysmorphic disorder. Body Image. 2004;1:113-25.

25. Castle DJ, Phillips KA, Dufresne RG Jr. Body dysmorphic disorder and cosmetic dermatology: more than skin deep. J Cosmet Dermatol. 2004;3:99-103.

26. Grant JE, Phillips KA. Recognizing and treating body dysmorphic disorder. Ann Clin Psychiatry. 2005;17:205-10.

27. Phillips KA, Menard W, Fay C. Gender similarities and differences in 200 individuals with body dysmorphic disorder. Compr Psychiatry. 2006;47:77-87.

28. Crerand CE, Phillips KA, Menard W, Fay C. Nonpsychiatric medical treatment of body dysmorphic disorder. Psychosomatics. 2005;46:549-55.

29. Phillips KA, Dufresne RG. Body dysmorphic disorder. A guide for dermatologists and cosmetic surgeons. Am J Clin Dermatol. 2000;1:235-43.

30. Hunter-Yates J, Dufresne RG Jr, Phillips KA. Tanning in body dysmorphic disorder. J Am Acad Dermatol. 2007;56(5 Suppl):S107-9.

31. Pope CG, Pope HG, Menard W, Fay C, Olivardia R, Phillips KA. Clinical features of muscle dysmorphia among males with body dysmorphic disorder. Body Image. 2005;2:395-400.

32. Ruffolo JS, Phillips KA, Menard W, Fay C, Weisberg RB. Comorbidity of body dysmorphic disorder and eating disorders: severity of psychopathology and body image disturbance. Int J Eat Disord. 2006;39:11-9.

33. Grant JE, Menard W, Phillips KA. Pathological skin picking in individuals with body dysmorphic disorder. Gen Hosp Psychiatry. 2006;28:487-93.

34. Hollander E, Kim S, Khanna S, Pallanti S. Obsessivecompulsive disorder and obsessive-compulsive spectrum disorders: diagnostic and dimensional issues. CNS Spectr. 2007;12(2 Suppl 3):5-13.

35. Buhlmann U, Cook LM, Fama JM, Wilhelm S. Perceived teasing experiences in body dysmorphic disorder. Body Image. 2007;4:381-5.

36. Phillips KA. Psychosis in body dysmorphic disorder. J Psychiatr Res. 2004;38:63-72.

37. Marazziti D, Giannotti D, Catena MC, Carlini M, Dell'Osso B, Presta S, et al. Insight in body dysmorphic disorder with and without comorbid obsessivecompulsive disorder. CNS Spectr. 2006;11:494-8.

38. American Psychiatric Association. Diagnostic and Statistical Manual of Mental Disorders, Fourth Edition, 
Text Revision. Washington, D.C.: American Psychiatric Association, 2000.

39. Bienvenu OJ, Samuels JF, Riddle MA, Hoehn-Saric R, Liang KY, Cullen BA, et al. The relationship of obsessivecompulsive disorder to possible spectrum disorders: results from a family study. Biol Psychiatry. 2000;48:287-93.

40. Koran LM, Abujaoude E, Large MD, Serpe RT. The prevalence of body dysmorphic disorder in the United States adult population. CNS Spectr. 2008;13:316-22.

41. Rief W, Buhlmann U, Wilhelm S, Borkenhagen A, Brahler $\mathrm{E}$. The prevalence of body dysmorphic disorder: a population-based survey. Psychol Med. 2006;36:877-85.

42. Bohne A, Wilhelm S, Keuthen NJ, Florin I, Baer L, Jenike MA. Prevalence of body dysmorphic disorder in a German college student sample. Psychiatry Res. 2002;109:101-4.

43. Altamura C, Paluello MM, Mundo E, Medda S, Mannu P. Clinical and subclinical body dysmorphic disorder. Eur Arch Psychiatry Clin Neurosci. 2001;251:105-8.

44. Aouizerate B, Pujol H, Grabot D, Faytout M, Suire K, Braud $\mathrm{C}$, et al. Body dysmorphic disorder in a sample of cosmetic surgery applicants. Eur Psychiatry. 2003; 18:365-8.

45. Zimmerman M, Mattia JI. Body dysmorphic disorder in psychiatric outpatients: recognition, prevalence, comorbidity, demographic, and clinical correlates. Compr Psychiatry. 1998;39:265-70.

46. Fontenelle LF, Telles LL, Nazar BP, de Menezes GB, do Nascimento AL, Mendlowicz MV, et al. A sociodemographic, phenomenological, and long-term follow-up study of patients with body dysmorphic disorder in Brazil. Int J Psychiatry Med. 2006;36:243-59.

47. Conroy M, Menard W, Fleming-Ives K, Modha P, Cerullo $\mathrm{H}$, Phillips KA. Prevalence and clinical characteristics of body dysmorphic disorder in an adult inpatient setting. Gen Hosp Psychiatry. 2008;30:67-72.

48. Hepburn S, Cunningham S. Body dysmorphic disorder in adult orthodontic patients. Am J Orthod Dentofacial Orthop. 2006;130:569-74.

49. Tignol J, Biraben-Gotzamanis L, Martin-Guehl C, Grabot D, Aouizerate B. Body dysmorphic disorder and cosmetic surgery: evolution of 24 subjects with a minimal defect in appearance 5 years after their request for cosmetic surgery. Eur Psychiatry. 2007;22:520-4.

50. Kittler JE, Menard W, Phillips KA. Weight concerns in individuals with body dysmorphic disorder. Eat Behav. 2007;8:115-20.

51. Ishigooka J, Iwao M, Suzuki M, Fukuyama Y, Murasaki M, Miura S. Demographic features of patients seeking cosmetic surgery. Psychiatry Clin Neurosci. 1998; 52:283-7.

52. Taqui AM, Shaikh M, Gowani SA, Shahid F, Khan A, Tayyeb SM, et al. Body Dysmorphic Disorder: gender differences and prevalence in a Pakistani medical student population. BMC Psychiatry. 2008;8:20.

53. Feusner JD, Yaryura-Tobias J, Saxena S. The pathophysiology of body dysmorphic disorder. Body Image. 2008;5:3-12.
54. Mathew SJ. PANDAS variant and body dysmorphic disorder. Am J Psychiatry. 2001;158:963.

55. Gabbay V, Asnis GM, Bello JA, Alonso CM, Serras SJ, O'Dowd MA. New onset of body dysmorphic disorder following frontotemporal lesion. Neurology. 2003;61:123-5.

56. Buhlmann U, McNally RJ, Wilhelm S, Florin I. Selective processing of emotional information in body dysmorphic disorder. J Anxiety Disord. 2002;16:289-98.

57. Gunstad J, Phillips KA. Axis I comorbidity in body dysmorphic disorder. Compr Psychiatry. 2003;44:270-6.

58. Bellino S, Zizza M, Paradiso E, Rivarossa A, Fulcheri M, Bogetto F. Dysmorphic concern symptoms and personality disorders: a clinical investigation in patients seeking cosmetic surgery. Psychiatry Res. 2006;144:73-8.

59. Phillips KA, Didie ER, Menard W. Clinical features and correlates of major depressive disorder in individuals with body dysmorphic disorder. J Affect Disord. 2007;97:129-35.

60. Pinto A, Phillips KA. Social anxiety in body dysmorphic disorder. Body Image. 2005;2:401-5.

61. Phillips KA, Pinto A, Jain S. Self-esteem in body dysmorphic disorder. Body Image. 2004;1:385-90.

62. Frare F, Perugi G, Ruffolo G, Toni C. Obsessive-compulsive disorder and body dysmorphic disorder: a comparison of clinical features. Eur Psychiatry. 2004;19:292-8.

63. Phillips KA, Pinto A, Menard W, Eisen JL, Mancebo M, Rasmussen SA. Obsessive-compulsive disorder versus body dysmorphic disorder: a comparison study of two possibly related disorders. Depress Anxiety. 2007;24:399-409.

64. Phillips KA, Pagano ME, Menard W. Pharmacotherapy for body dysmorphic disorder: treatment received and illness severity. Ann Clin Psychiatry. 2006;18:251-7.

65. Phillips KA. Placebo-controlled study of pimozide augmentation of fluoxetine in body dysmorphic disorder. Am J Psychiatry. 2005; 162:377-9.

66. Phillips KA. An open-label study of escitalopram in body dysmorphic disorder. Int Clin Psychopharmacol. 2006;21:177-9.

67. Neziroglu F, Cash TF. Body dysmorphic disorder: causes, characteristics, and clinical treatments. Body Image. 2008;5:1-2.

68. Buhlmann U, Reese HE, Renaud S, Wilhelm S. Clinical considerations for the treatment of body dysmorphic disorder with cognitive-behavioral therapy. Body Image. 2008;5:39-49.

69. Phillips KA, Dufresne RG. Body dysmorphic disorder. A guide for dermatologists and cosmetic surgeons. Am J Clin Dermatol. 2000;1:235-43.

ENDEREÇO PARA CORRESPONDÊNCIA / MAILING ADDRESS:
Luciana Archetti Conrado
Av. Pavão, 955 - CJ. $47 / 48$ - Moema
04516012São Paulo SP
luconrado@yaboo.com.br

ENDEREÇO PARA CORRESPONDÊNCIA / MAILING ADDRESS:

Av. Pavão, 955 - CJ. 47/48 - Moema

luconrado@yaboo.com.br

Como citar este artigo / How to cite this article: Conrado LA. Transtorno dismórfico corporal em dermatologia: diagnóstico, epidemiologia e aspectos clínicos. An Bras Dermatol. 2009;84(6):569-81. 